\title{
Análise Experimental da Eficácia do Método de Inspeção Semiótica para Avaliação de Resposta Emocional
}

\author{
Letícia Gabrielly Zacano da Silva \\ Universidade Federal de São Carlos - UFSCar \\ São Carlos - SP, Brasil \\ leticiazacano@ufscar.br
}

\author{
Vânia Paula de Almeida Neris \\ Universidade Federal de São Carlos - UFSCar \\ São Carlos - SP, Brasil \\ vania@dc.ufscar.br
}

\begin{abstract}
RESUMO
Em Interação Humano-Computador (IHC), avaliações ajudam a conhecer os desejos e problemas que usuários enfrentam interagindo com interfaces. Podem ser executadas avaliações empiricamente observando usuários e analiticamente com avaliadores inspecionando interfaces para encontrar problemas e sugerir melhorias. Avaliações podem investigar usabilidade, acessibilidade, comunicabilidade de sistemas e a resposta emocional de usuários. Na literatura existem alguns métodos para analisar emoções, como sinais fisiológicos. Eles são responsáveis por regular sistemas do organismo que acompanham emoções. A medição por sinais fisiológicos é cara e invasiva. O Método de Inspeção Semiótica para avaliação de respostas Emocionais (MISE) é uma alternativa barata e menos trabalhosa para avaliar o estado emocional. O levantamento da literatura executado encontrou 31 trabalhos que aferiram a eficácia de métodos analíticos e nenhum avaliou o estado emocional. O objetivo dessa proposta é aferir se o MISE é eficaz para avaliar a resposta emocional de usuários. Serão realizados dois experimentos, com usuários reais de interfaces e seus sinais fisiológicos e estudiosos em IHC aplicando o MISE, seus resultados serão comparados e a eficácia do MISE será aferida.
\end{abstract}

\section{PALAVRAS-CHAVE}

Emoções, métodos de avaliação, sinais fisiológicos, comparação experimental

\section{INTRODUÇÃO}

Dentro da área de Interação Humano-Computador (IHC) é investigado o design, a avaliação e a implementação de sistemas interativos [1]. Avaliações ajudam a conhecer os desejos e os problemas que os usuários enfrentam na interação com interfaces. Existem várias técnicas disponíveis para se coletar dados sobre interfaces e se fazer a análise da sua qualidade de uso. A análise da qualidade de Interfaces de Usuário (IU) pode ser realizada por meio de avaliações empíricas e analíticas [19]. As avaliações empíricas são aquelas que envolvem a observação de usuários ao interagirem com interfaces [28].

Permission to reproduce or distribute, in whole or in part, material extracted from this work, verbatim, adapted or remixed, as well as the creation or production from the content of such work, is granted without fee for non-commercial use, provided that the original work is properly credited.

IHC 2019 - Workshop de Teses e Dissertações, Outubro 21-25, 2019, Vitória, Brasil. In Anais Estendidos do XVIII Simpósio Brasileiro sobre Fatores Humanos em Sistemas Computacionais. Porto Alegre: SBC.

(C) 2019 by the author(s), in accordance with the terms of the Creative Commons Attribution-NonCommercial 4.0 International Public License (CC BY-NC 4.0).
As analíticas são aquelas que avaliadores inspecionam aspectos das interfaces com a intenção de encontrar problemas, geralmente relacionados a usabilidade, e sugerir melhorias [19]. A análise da qualidade de uso de IU, também pode avaliar aspectos como usabilidade, acessibilidade, comunicabilidade de sistemas e a resposta emocional de usuários. Enquanto os usuários interagem com uma IU podem ser despertados diferentes estados emocionais como, por exemplo, frustração por não conseguir alcançar um objetivo.

Para [24], emoção é definida como um episódio de alterações inter-relacionadas e sincronizadas por todos, ou quase todos, os subsistemas (componentes) do organismo em resposta a eventos de estímulo externo ou interno, para os principais interesses do organismo. Segundo [25], a avaliação emocional ideal avalia 5 componentes: avaliação cognitiva, reações fisiológicas, tendências comportamentais, expressão motora e sentimento subjetivo.

As reações fisiológicas (sinais fisiológicos) são responsáveis pela regulação dos sistemas do organismo que acompanham as emoções. A maioria dos instrumentos para a medição dos sinais fisiológicos, possuem um alto custo, são invasivos e necessitam de especialistas para a utilização de equipamentos complexos [11].

Os sinais fisiológicos podem ser medidos por meio de Resposta Galvânica (GSR) da pele, para a coleta da eletro condutividade da pele; Eletrocardiograma (ECG), para registrar batimentos cardíacos; Eletroencefalograma (EEG), para a coleta de atividade elétrica no cérebro, etc [5]. O Método de Inspeção Semiótica para avaliação de respostas Emocionais (MISE), foi criado como uma alternativa mais barata e menos trabalhosa, para a avaliação do estado emocional, pois, não necessita da participação de usuários para ser aplicado [22].

Um levantamento da literatura foi executado para elencar trabalhos que tinham como objetivo aferir a eficácia de métodos de avaliação de IU analíticos. Foram encontrados 31 trabalhos que realizaram comparações de métodos analíticos com empíricos, afim de aferir a eficácia. Em sua maioria os trabalhos analisaram métodos de usabilidade e nenhum deles analisou a resposta emocional de usuários. Até o momento a eficácia do MISE, não foi aferida.

A presente proposta de pesquisa, tem por objetivo realizar uma investigação experimental para aferir a eficácia do método MISE para avaliar a resposta emocional evocada por IU. Serão realizados dois experimentos, com usuários reais de interfaces e seus sinais fisiológicos e especialistas em IHC aplicando o MISE, seus resultados serão comparados e a eficácia do MISE será aferida. 


\section{CONCEITOS FUNDAMENTAIS}

\subsection{Avaliações Analíticas}

Segundo [8], a área de IHC estuda o design, a avaliação e a implementação de sistemas computacionais interativos para o uso humano, e os principais fenômenos associados a este uso. Os estudos que envolvem o design são relacionados a construir interfaces por meio de métodos, modelos e diretrizes. Os estudos que envolvem a avaliação são relacionados a avaliar a qualidade de um projeto de interface [19]. E os estudos relacionados a implementação envolvem o desenvolvimento do sistema computacional em si.

Nessa proposta, o foco será nos estudos de IHC relacionados as avaliações de interfaces. Interface e interação são termos muito utilizados quando o assunto é a avaliação de sistemas computacionais. Interface é toda parte do sistema com a qual o usuário mantém contato enquanto o utiliza, seja de forma física, perceptiva ou conceitual [14].

A Interação é o processo de comunicação entre pessoas e sistemas interativos [20]. É como uma conversa, onde em alguns momentos o usuário fala e o sistema ouve, interpreta e realiza uma ação, vice e versa. Em IHC, esse processo de comunicação é estudado principalmente do ponto de vista do usuário, observando suas ações e interpretações sobre as respostas do sistema por meio da interface [19].

Os métodos de avaliação analíticos não envolvem a participação direta do usuário, mas sim, experiências de uso potenciais e não reais. Para inspecionar uma interface, os avaliadores se colocam no lugar de um usuário com um determinado perfil, nível de conhecimento e experiência. Então, o avaliador tenta encontrar problemas que podem surgir quando os usuários interagirem com o sistema, e de que formas o sistema irá ajuda-los a resolver tais problemas [2].

Os métodos analíticos podem avaliar aspectos como: usabilidade, por meio da Avaliação Heurística; acessibilidade, por meio da Avaliação Simplificada de Acessibilidade (ASA); e a comunicabilidade de sistemas, por meio do Método de Inspeção Semiótica (MIS).

\subsection{Avaliações de Emoções}

As emoções estão presentes em nosso cotidiano em ações e pensamentos, mesmo que inconscientemente agimos segundo alguma emoção. Elas podem nos ajudar a avaliar situações como boas ou ruins $[1,15]$. Em IHC, as emoções estão relacionadas a experiência do usuário e a avaliação da qualidade de produtos. Estímulos como cores, forma, multimídia, entre outros, podem evocar emoções positivas ou negativas na interação do usuário com um sistema [5].

Na literatura é possível encontrar alguns métodos para avaliação e classificação das emoções [32]. Como, por exemplo, os métodos Think Aloud [27], Self-Assessment Manikin (SAM) [3], Emocards [21], Facial Action Coding System (FACS) [7], Dez Heurísticas da Emoção [5] e a abordagem híbrida de [31].

O Espaço Emocional Semântico (EES) de Scherer [25], é uma ferramenta para classificar emoções. Ele é dividido em quatro domínios emocionais que possuem polos positivos e negativos. Em cada domínio existem subdivisões, denominadas oitantes, onde são distribuídas emoções que permitem uma representação gráfica. Nessa pesquisa o EES será utilizado para classificar o estado emocional que as interfaces selecionadas despertam.

\subsection{MISE}

O MISE tem como base às dez classes de signos de [16, 17]. Por meio das definições dessas dez classes é possível classificar os elementos de interfaces. Na criação do MISE percebeu-se que algumas das classes são semelhantes. Com isso, o MISE as agrupa em três grupos de signos: icônicos, indicativos e informativos. Considerando as características de cada grupo, enquanto se inspecionam as interfaces, são feitas questões específicas que levam a associação de uma ou mais dimensões do EES [22].

Basicamente a aplicação do MISE consiste em selecionar IU e seus elementos para realizar uma análise semiótica e entender a função dos elementos em seu contexto. A seguir é explicado brevemente a execução das quatro etapas do MISE.

2.3.1 Etapa 1-Seleção de interfaces e elementos a serem analisados. Na primeira etapa, os avaliadores devem definir o perfil dos usuários; descrever a tarefa principal, sub tarefas e a resposta do sistema a essas tarefas; selecionar as interfaces e os elementos ou eventos de interface, que sejam relevantes às tarefas.

2.3.2 Etapa 2-Categorização semiótica dos elementos de interface. Na segunda etapa, é feita a categorização dos elementos selecionados de acordo com os três grupos de signos: Icônicos, signos que apresentam similaridades com seu objeto; indicativos, signos que indicam uma causa e/ou consequência sobre o objeto; e Informativos: signos que representam uma ideia. A categorização é feita por um diagrama, que possui perguntas, que ao serem respondidas levam a um grupo de signo que categoriza o elemento analisado.

2.3.3 Etapa 3 - Identificação das dimensões e os oitantes do EES. $\mathrm{Na}$ terceira etapa, cada grupo de signos possuem perguntas que foram elaboradas com base nas quatro dimensões do EES e nas características dos signos, com a intenção de associá-los aos oitantes.

2.3.4 Etapa 4-Análise de incidências e consolidação dos resultados. Na quarta e última etapa, é feita a análise de incidências dos oitantes selecionados para cada elemento avaliado nas etapas anteriores, de acordo com o conjunto de questões. As incidências são identificadas pelo cálculo da moda para verificar qual oitante aparece com mais frequência. A moda não necessariamente irá resultar em apenas um oitante. Ao final da análise de incidências, se houver mais de um avaliador, é feita a consolidação dos resultados de todos os avaliadores.

\section{SOLUÇÃO PROPOSTA}

A partir do levantamento do estado da arte sobre a eficácia de métodos de avaliação de IU observou-se que a maioria dos trabalhos compararam métodos analíticos e empíricos analisando o aspecto de usabilidade. Essa proposta busca comparar métodos que analisem a resposta emocional de usuários. O método empírico que infere o estado emocional por meio de sinais fisiológicos, adotado nessa pesquisa, foi escolhido por ser um dos componente 5 componentes propostos por [25] para uma avaliação emocional ideal.

E por conta de que os sensores fisiológicos a serem utilizados, terem sidos estudados na proposta de [6] que por meio da criação 
de um data set classifica emoções de acordo com o EES de Scherer. A escolha do MISE para a comparação proposta, justifica-se pelo fato de ele ser um método relativamente novo e ainda não ter sido investigada sua eficácia.

Justifica-se também por ser uma alternativa mais barata para a inferência da resposta emocional de usuários. Ambos os métodos de avaliação selecionados, resultarão em um conjunto de emoções do EES de Scherer. Os métodos serão comparados afim de aferir a eficácia do MISE em relação ao método empírico e serão feitas relações entre o custo e a eficiência dos métodos.

\section{TRABALHOS RELACIONADOS}

O objetivo do Mapeamento Sistemático (MS) foi, encontrar o estado da arte sobre a comparação da eficácia de métodos de avaliação de IU. Os primeiros processos de MS, foram realizados no período de março a maio de 2019. Nessa fase inicial, consideraram-se os passos propostos por [18], para sua execução. Foram definidas e aprimoradas as seguintes strings de busca:

("analytical method"OR "inspection method"OR "evaluation method "OR "interface evaluation" $O R$ "empirical method"OR "empirical evaluation") AND ("experimental comparison"OR "experimental evaluation"OR "experimental analysis") AND ("human computer interaction"OR "computer human interaction")

("inspection method"OR "analytical methods"OR "empirical evaluation"OR "empirical method"OR "evaluation method"OR "interface evaluation") AND ("comparison"OR "analysis"OR "assess"OR "comparative" $O R$ "evaluate" $O R$ "evaluation"OR "validation") AND ("user interface" $O R$ "user test")

Nas seguintes bases de dados: ACM Digital Library, IEEE Xplore Digital Library e SCOPUS Elsevier. A busca referente a primeira string, foi executada até sua fase final e retornou um total de 248 trabalhos. Após realizar todo o processo de extração de dados, que envolve: ler títulos e resumos, aplicar os critérios de inclusão e exclusão e analisar o trabalho completo. Foram aceitos apenas 2 trabalhos. O que é um número pequeno para um MS.

Considerando o baixo número de trabalhos retornados na primeira busca e a quantidade de trabalhos aceitos, a segunda string de busca foi executada. Foram retornados um total de 4.383 trabalhos. Logo no início do processo de análise dos trabalhos foi possível notar que muitos dos ali presentes, não se relacionavam com o objetivo do MS. Então foi tomada a decisão de interromper o processo de busca.

Após várias mudanças nas strings de busca, além das apresentadas, observou-se que mais da metade dos trabalhos retornados eram sobre a aplicação de métodos de avaliação em interfaces. E não sobre a avaliação de métodos de avaliação de IU. Por esse motivo, foi estudado o procedimento Snowballing, de [30], para aplicá-lo nesse projeto de pesquisa.

O procedimento de MS Snowballing, ou em português "bola de neve", é realizado considerando a lista de referências e citações de um artigo, inicialmente selecionado, para encontrar outros artigos que se relacionam com ele [30]. Para iniciar o procedimento é necessário selecionar um conjunto inicial de artigos que são relacionados com o objetivo do MS. O procedimento foi realizado entre junho e julho de 2019.
Para selecionar o conjunto inicial é indicado realizar buscas no Google Scholar, para não ocorrer o viés de ter selecionado uma base de dados específica. A string, deve ser formulada a partir das palavras-chave e questão de pesquisa. Para essa pesquisa foi definida a seguinte string de busca: (assessing AND inspection method AND HCI).

Após realizar os procedimentos de snowballing para trás, que consiste em encontrar novos artigos a partir da lista de referências de um artigo já aceito. E o snowballing para frente, que consiste em encontrar novos artigos a partir das citações de um artigo já incluído. Foram selecionados 31 trabalhos relacionados ao objetivo do MS.

Trinta dos 31 trabalhos incluídos realizaram comparações de métodos de avaliação relacionados ao aspecto de usabilidade, como o de [9]. Outros trabalhos aos aspectos de acessibilidade, como o de [4] e comunicabilidade, como o de [23]. Não foram encontrados trabalhos que comparassem métodos de avaliação relacionados ao aspecto emocional.

O trabalho de [12] realizou uma comparação experimental da eficácia e eficiência de dois métodos de usabilidade, o teste de usabilidade e a avaliação heurística. $\mathrm{Na}$ análise dos resultados, foram considerados como medidas de eficácia aspecto como: número de problemas identificados; gravidade de problemas; tempo para encontrar problemas; e tipos de problemas.

Trabalhos como os de [10, 13, 26, 29], de maneira semelhante ao trabalho de [12], comparam métodos de avaliação relacionados ao aspecto de usabilidade. Grande parte dos trabalhos encontrados, realizaram a comparação de métodos analíticos com empíricos. Alguns autores comentaram que a combinação de métodos empíricos com analíticos podem gerar avaliações mais enriquecedoras e completas.

Com a conclusão do MS realizado foi possível identificar alguns critérios de comparação de eficácia de métodos de avaliação, de usabilidade, acessibilidade e comunicabilidade, que também podem ser utilizados para comparar métodos que avaliem a resposta emocional. Como por exemplo a eficiência, o custo, a satisfação e a confiabilidade dos métodos.

\section{METODOLOGIA}

Serão realizados dois experimentos, um com o método analítico MISE e outro com o método empírico que utiliza sensores fisiológicos. Atendendo ao rigor ético e científico o projeto de pesquisa foi encaminhado e aprovado pelo Comitê de Ética em Pesquisa em Seres Humanos (CEP), da UFSCar, sob o CAAE: 16850419.6.0000.5504.

O experimento com o MISE será aplicado por um conjunto de 12 estudiosos em IHC. Como o MISE é relativamente novo, ainda não existem avaliadores especialistas em sua aplicação. Com isso, será oferecido um treinamento para sua aplicação. Ao final do treinamento os estudiosos aplicarão o MISE em três interfaces: um site de compras, um aplicativo de agenda e um jogo. O critério de inclusão nesse experimento é: pessoas que tenham conhecimento na área de IHC.

O experimento com o método empírico, será realizado com 30 usuários comuns de interfaces. Enquanto os usuários estiverem interagindo com às três interfaces selecionadas, eles terão seus sinais fisiológicos e sentimentos subjetivos coletados. Os critérios 
de inclusão nesse experimento são: idade maior que 18 anos, ensino médio completo e pessoas que possuam qualquer tipo de notebook e celular com sistema operacional Android em qualquer versão. Os critérios de exclusão são: problemas cardíacos e hiperidrose. Os sinais fisiológicos serão coletados pelos sensores fisiológicos ECG, GSR, EEG.

\subsection{Análise dos dados}

Após a coleta dos dados do método empírico ,os sinais fisiológicos passarão por pré-processamento e normalização dos sinais. Os sinais fisiológicos classificados em emoções, serão mapeados no Espaço Emocional Semântico de Scherer no oitante correspondente. Com a consolidação dos resultados do MISE, aplicado por estudiosos em IHC, serão retornados um ou mais oitantes presentes no Espaço Emocional Semântico de Scherer. Considerando como métrica de eficácia o método empírico, se a aplicação do MISE resultar em um conjunto de emoções coincidentes ou semelhantes aos do método empírico, então ele será considerado eficaz para inferir o estado emocional evocado por uma IU.

\section{CONTRIBUIÇÕES ESPERADAS}

Como resultado e contribuição dessa proposta de pesquisa, pretendese comprovar se o MISE é tão eficaz quanto os sensores fisiológicos para inferir um estado emocional despertado por IU. Também será feita a comparação dos métodos, empírico e analítico, na intenção de coletar informações de eficiência e custo-benefício, como, tempo para aplicação dos métodos, recursos necessários (como habilidades, local e participantes), gastos (com equipamentos) e dificuldades na aplicação dos métodos. Depois de validados esses critérios de comparação poderão contribuir com pesquisas que avaliem métodos de avaliação de resposta emocional.

\section{REFERÊNCIAS}

[1] Anshu Agarwal and Andrew Meyer. 2009. Beyond usability: evaluating emotional response as an integral part of the user experience. In CHI'09 Extended Abstracts on Human Factors in Computing Systems. ACM, 2919-2930.

[2] Simone Barbosa and Bruno Silva. 2010. Interação humano-computador. Elsevier Brasil.

[3] Margaret M Bradley and Peter J Lang. 1994. Measuring emotion: the selfassessment manikin and the semantic differential. fournal of behavior therapy and experimental psychiatry 25, 1 (1994), 49-59.

[4] Giorgio Brajnik. 2008. A comparative test of web accessibility evaluation methods In Proceedings of the 10th international ACM SIGACCESS conference on Computers and accessibility. ACM, 113-120.

[5] Eva De Lera and Muriel Garreta-Domingo. 2007. Ten emotion heuristics: guidelines for assessing the user's affective dimension easily and cost-effectively. In Proceedings of the 21st British HCI Group Annual Conference on People and Computers: HCI... but not as we know it-Volume 2. BCS Learning \& Development Ltd., 163-166.

[6] Isaque Elcio de Souza. 2019. Classificação de sinais fisiológicos para inferência do estado emocional de usuários. Dissertação. Master's thesis. Universidade Federal de São Carlos, São Carlos.

[7] Paul Ekman, Wallace V Friesen, and Joseph C Hager. 2002. Facial action coding system. A Human Face, Salt Lake City (2002), 77-254

[8] Thomas T Hewett, Ronald Baecker, Stuart Card, Tom Carey, Jean Gasen, Marilyn Mantei, Gary Perlman, Gary Strong, and William Verplank. 1992. ACM SIGCHI curricula for human-computer interaction. ACM.

[9] Reza Khajouei, Sadrieh Hajesmaeel Gohari, and Moghaddameh Mirzaee. 2018 Comparison of two heuristic evaluation methods for evaluating the usability of health information systems. Fournal of biomedical informatics 80 (2018), 37-42.

[10] Reza Khajouei, Misagh Zahiri Esfahani, and Yunes Jahani. 2017. Comparison of heuristic and cognitive walkthrough usability evaluation methods for evaluating health information systems. Fournal of the American Medical Informatics Association 24, e1 (2017), e55-e60.
[11] GFG Laurans, Pieter MA Desmet, and Paul PM Hekkert. 2009. Assessing emotion in interaction: some problems and a new approach. In Proceedings of the 4th International Conference on Designing Pleasurable Products and Interfaces, DPPI'09, Compiegne, Oct. 2009. Universite de Technologie de Compiegne.

[12] Martin Maguire and Paul Isherwood. 2018. A Comparison of User Testing and Heuristic Evaluation Methods for Identifying Website Usability Problems. In International Conference of Design, User Experience, and Usability. Springer, 429438.

[13] Rolf Molich and Joseph S Dumas. 2008. Comparative usability evaluation (CUE-4). Behaviour \& Information Technology 27, 3 (2008), 263-281.

[14] Thomas P Moran. 1981. The command language grammar: A representation for the user interface of interactive computer systems. International journal of man-machine studies 15, 1 (1981), 3-50.

[15] Donald A Norman. 2004. Emotional design: Why we love (or hate) everyday things. Basic Civitas Books.

[16] CS PEIRCE. 2005. Semiótica. 2005.

[17] Charles Sanders Peirce. 1974. Collected papers of charles sanders peirce. Vol. 2. Harvard University Press.

[18] Kai Petersen, Robert Feldt, Shahid Mujtaba, and Michael Mattsson. 2008. Systematic mapping studies in software engineering.. In Ease, Vol. 8. 68-77.

[19] Raquel Oliveira Prates and Simone Diniz Junqueira Barbosa. 2003. Avaliação de interfaces de usuário-conceitos e métodos. In fornada de Atualização em Informática do Congresso da Sociedade Brasileira de Computação, Capítulo, Vol. 6. 28.

[20] Jenny Preece, Yvonne Rogers, Helen Sharp, David Benyon, Simon Holland, and Tom Carey. 1994. Human-computer interaction. Addison-Wesley Longman Ltd.

[21] Karen Reijneveld, Michiel De Looze, Frank Krause, and Pieter Desmet. 2003. Measuring the emotions elicited by office chairs. In Proceedings of the 2003 international conference on Designing pleasurable products and interfaces. ACM, 6-10.

[22] Maria Carolina de Souza Santos et al. 2016. Um método analítico para avaliação de respostas emocionais na Interação Humano-Computador. (2016).

[23] Rafael Dias SANTOS and Bruno Santana da SILVA. 2018. Comparação de Métodos de Avaliação de IHC sob a Perspectiva do Autor da Interface. (2018).

[24] K R Scherer. 2001. Appraisal processes in emotion: theory, method, research. Appraisal considered as a process of multilevel sequential checking 4, 4 (2001), 92-120.

[25] Klaus R Scherer. 2005. What are emotions? And how can they be measured? Social science information 44, 4 (2005), 695-729.

[26] Ashok Sivaji, Azween Abdullah, and Alan Giffin Downe. 2011. Usability testing methodology: Effectiveness of heuristic evaluation in E-government website development. In 2011 Fifth Asia Modelling Symposium. IEEE, 68-72.

[27] MW Van Someren, YF Barnard, and JAC Sandberg. 1994. The think aloud method: a practical approach to modelling cognitive. Citeseer.

[28] Heloisa Vieira and Maria Cecília C Baranauskas. 2003. Design e avaliação de interfaces humano-computador. Creative Commons, Brasil.

[29] Steve Wallace, Adrian Reid, Jin-Su Kang, and Daniel Clinciu. 2013. A comparison of the usability of heuristic evaluations for online help. Information Design fournal 20, 1 (2013), 58-68.

[30] Claes Wohlin. 2014. Guidelines for snowballing in systematic literature studies and a replication in software engineering. In Proceedings of the 18th international conference on evaluation and assessment in software engineering. Citeseer, 38.

[31] R.A.C. Xavier and V.P. De Almeida Neris. 2012. A hybrid evaluation approach for the emotional state of information systems users. ICEIS 2012 - Proceedings of the 14th International Conference on Enterprise Information Systems 3 (01 2012), 45-53.

[32] Rogério Aparecido Campanari Xavier. 2013. Uma abordagem híbrida para a avaliação da experiência emocional de usuários. (2013). 